\title{
USER-GENERATED CONTENT EM SERVIÇOS: UMA ANÁLISE DO AEROPORTO INTERNACIONAL DE GUARULHOS (GRU)
}

USER-GENERATED CONTENT IN SERVICES: AN ANALYSIS OF THE INTERNATIONAL AIRPORT OF GUARULHOS (GRU)

\author{
USER-GENERATED CONTENT EN SERVICIOS: ANÁLISIS DEL AEROPUERTO \\ INTERNACIONAL DE GUARULHOS (GRU)
}

\section{Ana Lúcia Borella Guido}

Pontifícia Universidade Católica de São Paulo - PUC/SP Doutoranda no Curso de Pós-Graduação em Administração Mestre em Administração (PUC/SP) analucia_borella@uol.com.br

\section{Belmiro do Nascimento João}

Pontifícia Universidade Católica de São Paulo - PUC/SP Coordenador do Curso de Doutorado no programa de Pós-Graduação em Administração Doutor em Comunicação e Semiótica (PUC/SP) Mestre em Administração (PUC/SP) bjoao@pucsp.br

Data de Submissão: 28/06/2016 Data de Aprovação: 21/10/2016

RESUMO: A principal inovação no setor aeroportuário brasileiro é a gestão da qualidade de serviços, após a privatização do setor. O objetivo desta pesquisa é analisar comentários (em língua inglesa) de usuários que avaliaram os serviços no Aeroporto Internacional de Guarulhos (GRU) colhidos entre 07/11/2011 e 18/05/2014. Sua importância está em usar a linguagem como análise na perspectiva dos usuários (service encounter). Para isso utiliza-se a análise de conteúdo além de regressão. Esta 
pesquisa, avaliará o poder da palavra para analisar a satisfação do cliente em relação ao serviço prestado, referente a três categorias: tempo de espera nas filas (queuing), instalações (facilities) e limpeza no ambiente (cleanline). Em complementação, os usuários podem recomendar, ou não, o aeroporto para outros viajantes. Resultados apontam que nos comentários positivos, a situação do aeroporto é aceitável, porém seguida de críticas. As variáveis de maior relevância são: instalações e tempo de espera nas filas. O que há de novo nesta análise é a dinâmica criada pela utilização de ferramentas de tecnologia da informação e a geração de conteúdo que pode ser utilizado para a co-criação, melhoria de qualidade ou redução de falhas em serviços.

PALAVRAS CHAVE: user-generated content (UGC), serviços, análise de conteúdo

ABSTRACT: The most remarkable innovation in the Brazilian airport industry is the services quality management, after the sector privatization. The purpose of this research is to analyze users' comments (in English) who evaluated services at Guarulhos International Airport (GRU), between 07/11/2011 and $05 / 18 / 2014$. Its importance is based on using the wording as an analysis on service encounter. The method used was content analysis as well as regression. This study is going to evaluate through that the power of words to analyze customer's satisfaction related to the services provided in three different categories: queuing, facilities and cleanline. In addition, the users may (or may not) recommend the airport to other travelers. The findings show that when comments are positive, the airport situation is acceptable but followed by criticism. The most relevant variables are: facilities and cleanline. What is new in this analysis is the dynamic process created by the use of Information Technology and content generation, which can be used on co-creation, quality improvement, and the reduction of services failures.

KEY WORDS: user-generated content (UGC), services, content analysis

RESUMEN: La principal innovación en el sector aeroportuario brasileño es la gestión de servicios de calidad, después de la privatización del sector. El objetivo de esta investigación es analizar los comentarios (en Inglés) de usuarios que clasificaron a los servicios en el Aeropuerto Internacional de Guarulhos (GRU) recogidos entre 07/11/2011 y 18/05/2014. Su importancia radica en el uso de la lengua como el análisis desde la perspectiva de los usuarios (service encounter). Para ello utilizamos el análisis de contenido, así como la regresión. Esta investigación evaluará la capacidad de hablar para analizar la satisfacción del cliente con el servicio, en referencia a tres categorías: el tiempo de espera en las colas (queuing), instalaciones (facilities) y la limpieza del medio ambiente (cleanline). Al finalizar, los usuarios pueden recomendar o no el aeropuerto a otros viajeros. Los resultados indican que las críticas positivas, la situación en el aeropuerto es aceptable, pero entonces crítico. La mayoría de las variables relevantes son: instalaciones y el tiempo de espera en las colas. ¿Qué hay de nuevo en este análisis es la dinámica creada por el uso de herramientas de tecnología de la información y la creación de contenido que puede ser utilizado para la co-creación, mejora de la calidad o la reducción de brechas en los servicios.

PALABRAS CLAVE: user-generated content (UGC). Servicios. Análisis de contenido

INTRODUÇÃO

A decisão de empregar, ou não, o uso da linguagem do cliente pode causar impacto de como o provedor de serviço percebe seu cliente (Van Vaerenbergh \& Holmqvist, 2014). A interação conjunta com o 
consumidor é a maneira pela qual a organização emprega a co-criação de valor com clientes (Grönroos \& Voima, 2013), tornando a gestão desta interação importante para as organizações. Estas devem considerar as preferências de linguagem do cliente. É necessário explorar a influência do uso da linguagem na percepção do cliente.

A prestação de serviços constrói bases sólidas de lealdade do cliente e cria vantagem competitiva para a empresa, dificultando a replicação do desempenho humano estabelecida entre uma corporação e seus consumidores (Lovelock \& Wirtz, 2010), sendo importante entender o serviço através da realidade do cliente (Shostack, 1984).

A principal inovação no setor aeroportuário brasileiro é a gestão da qualidade de serviços advinda da privatização do setor. O objetivo desta pesquisa é analisar comentários (em língua inglesa) de usuários que avaliaram os serviços no Aeroporto Internacional de Guarulhos (GRU). Sua importância está em usar a linguagem como análise e nos métodos inovadores de pesquisa.

Considerando-se que a principal porta de entrada no país é o aeroporto internacional de São Paulo este estudo oferece um panorama geral das condições estruturais do local na perspectiva dos usuários (service encounter). Analisa-se a opinião destes indivíduos referente a três categorias de serviços prestados pelo aeroporto: tempo de espera nas filas (queuing), instalações (facilities) e limpeza no ambiente (cleanline). Em complementação, os usuários podem recomendar, ou não, o aeroporto para outros viajantes além de atribuírem um ranking. A coleta das informações compreende o período de dois anos e sete meses com início em 07/11/2011 e término em 18/05/2014 e a metodologia aplicada é a análise de conteúdo complementada por análise estatística.

Esta pesquisa está assim dividida, além desta introdução, referencial teórico fortemente baseado em Holmqvist e Grönroos destacando o caráter estratégico dos serviços, conteúdo gerado pelo cliente (UGC), co-criação; metodologia, destacando-se a análise de conteúdo (Bardin, 2011); análise dos resultados; análise estatística e considerações finais além das referências.

Desta forma, esta pesquisa, avaliará o poder da palavra escrita (voz) para analisar a satisfação do cliente em relação ao serviço prestado. 


\section{O CARÁTER DINÂMICO DOS SERVIÇOS}

A estratégia de negócios corresponde a um conjunto de atividades e de decisões que determina os produtos e serviços que uma organização produz e o setor (indústria) em que atua, assim como seus concorrentes diretos ou potenciais, elos na cadeia de valores, seus fornecedores e clientes, além dos objetivos de longo prazo. Essa visão é compartilhada pela perspectiva da organização industrial (Estratégia-Concorrência-Desempenho) cujo representante mais conhecido são os trabalhos de Porter (1980, 1981, 1985, 1996). Empresas líderes globais em seus setores, como a Sueca Ikea, oferecem um número de serviços extras que seus competidores não possuem (Porter, 1996). Modelos de estratégia como o Modelo Delta (Hax, 2010) propõe que o uso de serviços integrados a produtos pode levar a um bloqueio dos competidores (lock-in). Treacy e Wiersema (1995) apontam a importância dos serviços e as expectativas do consumidor. Os autores apresentam um modelo com três estratégias genéricas de vantagem competitiva baseadas na excelência operacional, na liderança de produto e na intimidade com o consumidor. Esta última exige ofertas feitas sob medida, flexíveis e que empoderem os funcionários mais próximos aos clientes.

Define-se serviço como a aplicação especializada de competências (conhecimento e habilidade) por meio de ações, processos e desempenho, em benefício de outras entidades (potenciais clientes) a fim de cultivar e manter as relações com seus clientes, ou seja, o objetivo é avaliar a aceitação do mercado para melhorar a oferta e a prestação dos serviços aos clientes, criando propostas de valor específicas a cada necessidade (Vargo \& Lusch, 2004). Quanto às suas características, serviços são relativamente intangíveis, produzidos e consumidos simultaneamente, e geralmente menos padronizados do que bens (Berry, 1980; Shostack, 1984).

A prestação de serviços está intimamente relacionada às atividades humanas, principalmente aos funcionários de front-end (ou de linha de frente) que 
estabelecem o contato direto entre empresa e consumidor, seja por intermédio das mídias sociais, e-mail, telefone, atendimento de balcão, etc. Para uma empresa ser bem-sucedida na prestação de serviços é fundamental: contratar pessoas certas treiná-las, dar autonomia para tomada de decisões, organizá-las em equipes, motivá-las e recompensá-las pelo bom desempenho apresentado (Lovelock \& Wirtz, 2010). A interação entre o cliente final e o pessoal de atendimento do serviço sofre grande influência, tanto do tipo, como sobre a qualidade do serviço prestado para o cliente (Bitner, 1990; Grönroos, 2008). Esta relação entre a interação do pessoal de atendimento com o cliente, o tipo e a qualidade do serviço prestado é analisada por Lovelock e Wirtz (2010) em três categorias de ciclos de prestação de serviços, a saber: fracasso, mediocridade e sucesso, sendo os dois primeiros viciosos e apenas o de Sucesso virtuoso, onde ocorre a excelência do atendimento.

O consumidor tem um papel central em serviços. Surprenant e Solomon (1987) definem o serviço sob a perspectiva do cliente como uma interação mútua entre clientes e o pessoal de serviços. O papel do cliente na co-criação de valor em serviços tem sido enfatizado (Grönroos, 2008; Payne, Storbacka \& Frow, 2008). Bitner, Booms e Tetreault (1990) em um estudo utilizando a técnica do incidente crítico para a análise de falhas sob a perspectiva do cliente os autores coletaram 700 incidentes em companhias aéreas, hotéis e restaurantes, sendo estes categorizados em eventos particulares propuseram a sua classificação em três grupos diferentes. 1) falhas decorrentes de respostas dos funcionários a problemas no sistema de prestação de serviço; 2) falhas originárias de respostas dos funcionários a necessidades e pedidos específicos de clientes e 3) falhas decorrentes de atitudes imprevisíveis e não desejadas do pessoal de contato. De modo idêntico Treacy e Wiersema (1995) relatam a questão das falhas em serviços ao exemplificar a entrega de uma encomenda pela FedEx como muito positiva em contraste com as bagagens pela Delta, American e United Airlines.

Embora diversos métodos, ferramentas e instrumentos (por exemplo: Método Taguchi; poka-yoke - dispositivo contra falhas) (Fitzsimmons \& Fitzsimmons, 2014) sejam de conhecimento amplo o controle sobre falhas em serviços, muitos específicos de um setor, impede uma ação mais pontual na prevenção 
ou mesmo na recuperação de falhas (Stewart \& Chase, 1999). Chase e Stewart (1994) apresentam que os erros em serviços podem originar-se tanto da parte do prestador de serviço quanto da parte do cliente.

Novas formas organizacionais ou novos modelos de negócios surgem a todo o momento em um ambiente dinâmico. Desde a segunda metade da década de 90 vemos modelos organizacionais impactados pelas tecnologias de rede. Novos modelos de negócios como o Uber e airbnb.com são basicamente serviços, baseados em tecnologia, mas serviços. Muitos dos serviços do mundo físico migraram para uma constelação de empresas no mundo virtual como podemos ver.

The leading companies in the online travel agency industry are Expedia, Travelocity (which owns Lastminute.com), Orbitz, Priceline, Cheaptickets and a host of others. The online agents compete both with traditional travel agents (American Express, Thomas Cook, Carlson) and direct online sales by airlines, cruise lines and car rental companies. Their biggest business is selling airline tickets, where they employ the services of computerized airline reservation systems such as Sabre, Amadeus, Worldspan and Galileo. (Grant, 2010, p.92)

Ainda sob a perspectiva do marketing de serviços é importante entender o serviço através da realidade do cliente (Shostack, 1984), e também compreender os comportamentos e práticas de prestadores de serviços que constroem ou destroem a confiança dos consumidores, além de identificar os mecanismos que convertem a confiança do consumidor em valor, e lealdade em trocas relacionais (Sidershmukh, Singh \& Sabol, 2002). A prestação de serviço constrói bases sólidas de lealdade do cliente e cria vantagem competitiva para a empresa, pois se torna extremamente difícil para os concorrentes replicar o bom desempenho humano estabelecido em uma corporação (Lovelock \& Wirtz, 2010).

\section{A PALAVRA NAS ORGANIZAÇÕES E O CONTEÚDO GERADO PELO CLIENTE}

A decisão em empregar, ou não, o uso da linguagem do cliente pode ter um impacto significativo de como o provedor de serviço percebe seu cliente (Van Vaerenbergh \&, Holmqvist, 2014). A literatura de gestão e marketing 
de serviços indica que serviços dependem das interações das organizações como os clientes (Grönroos, 1990; Surprenant \& Solomon, 1987), e que o papel do prestador de serviços é muitas vezes confundido com o próprio serviço (Shostack, 1984).

Apesar desta ênfase na interação entre prestador de serviço e clientes, pesquisas recentes em serviços começam a questionar a influência da linguagem quando o pessoal de serviços e o cliente não falam o mesmo idioma (Holmqvist \& Grönroos, 2012). Isso é um fato surpreendente, dado que mais da metade dos países do mundo são multilíngues, e mais que a metade dos consumidores no mundo fala mais de um idioma (Luna \& Peracchio, 2001).

A interação conjunta com o consumidor é a maneira pela qual a organização emprega a co-criação de valor com os seus clientes (Grönroos \& Voima, 2013), sendo assim a gestão desta interação é de extrema importância para as organizações. Entretanto a tarefa de recrutar profissionais de serviços com competência linguística requerida e executando uma força de trabalho multilíngues é um desafio adicional para a gerência na organização (Harzing \& Pudelko, 2013; Piekkari, Welch, Welch, Peltonen \& Vesa, 2013). Mesmo a cocriação de serviços pode apresentar seu lado sóbrio (Heidenreich, Wittkowski, Handrich, \& Falk, 2014) dada a complexidade da relação de serviço.

Enquanto fundamental para toda a comunicação, a linguagem não é somente ferramenta funcional, clientes atribuem um forte valor emocional quando se expressam utilizando seu idioma nativo (Puntoni, De Langhe \& Van Osselaer, 2009).

É imprescindível que as organizações considerem as multifacetadas preferências de linguagem do cliente. Se por um lado, clientes de países nãoanglófonos podem apreciar o uso da língua inglesa (Spielmann \& Delvert, 2014), por outro, até mesmo os clientes capazes de falarem fluentemente uma segunda língua podem recusar-se enfaticamente a participar em um serviço prestado nesse idioma (Holmqvist, 2011), ou podem demonstrar um comportamento negativo se a organização não oferecer o serviço na linguagem desejada pelo cliente (Van Vaerenbergh \& Holmqvist, 2013; 2014). 
Independente do fator emocional, cada cliente tem a sua própria forma de expressão da linguagem. Pesquisas sobre marcas evidenciam que o uso da linguagem influencia a autenticidade percebida da marca pelo consumidor (Salciuviene, Ghauri, Streder, \& De Mattos, 2010), mas a questão de como o uso da linguagem influencia as percepções do cliente de serviço ainda necessitam ser exploradas.

Adiciona-se a essa complexidade o fato de que alguns clientes, que se sentem perfeitamente satisfeitos em trocar o idioma em certos serviços, demonstram uma forte resistência em mudar de idioma para outros serviços (Holmqvist \& Van Vaerenbergh, 2013). O uso da linguagem em serviços é uma questão complexa, que o marketing começa a investigar e a tatear.

Um desafio é o UGC (Fitzsimmons \& Fitzsimmons, 2014). O exemplo dado pelos autores é o da construção da enciclopédia Wikipedia.com por uma comunidade virtual assim como o da Craigslist.com, um sítio de classificados. Dennhardt (2014) destaca o papel da geração de conteúdo para a gestão do Branding e como os usuários e comunidades gerenciam marcas assim como as mídias sociais e a forte influência desta na formação de opinião que influencia a decisão de compra. Smith, Fischer e Yongjian (2012) destacam diferenças entre o YouTube, Facebook e Twitter no UGC relacionado a marcas.

De acordo com o Content Marketing Institute (2016), 86\% dos negócios usam do marketing de conteúdo e 78\% das empresas de B2C incorporaram UGC em 2015. O UGC emergiu como o novo "obrigatório" em estratégias de marketing, pois melhora o desempenho em todos os canais do marketing digital.

Segundo Shirky (2008) o UGC caracteriza-se quando usuários criam e compartilham mídia com outros, de maneira espontânea, isto é, sem a supervisão de profissionais. Kaplan \& Haenlein (2010), além de corroborarem com esta característica, acrescentam outras: o UGC contempla várias formas de conteúdo de mídia publicamente disponíveis (sites de acesso público, ou redes sociais acessíveis a um grupo seleto), devem ser elaboradas por usuários finais, fora das rotinas e práticas profissionais. Shirky (2008) conclui que a ideia do conteúdo gerado pelo usuário transcende a teoria pessoal das capacidades criativas, tornando-se uma teoria social de relações com a mídia. 
Alguns estudos específicos sobre o compartilhamento de experiências e impressões de viagem apresentam conclusões semelhantes: o UGC influencia outros usuários e é um desafio para os prestadores de serviço em turismo. Segundo Blackshaw \& Nazzaro (2006) estes profissionais enfrentam uma concorrência feroz no ambiente online, devido a quantidade de mídias e redes sociais que versam sobre o assunto (turismo, viagens, etc.), compartilham conteúdo, e podem influenciar a escolha, afetar o julgamento, e até a lealdade dos indivíduos que buscam informações sobre destinos de viagens.

Na abordagem de Xiang \& Gretzel (2010), apesar dos autores empregarem o termo modificado para CGC (consumer-generated content), confirmam a crescente importância dos UGCs para compartilhar informações sobre viagens, além de evidenciarem os desafios enfrentados pelos provedores tradicionais de turismo em fornecerem informações, devido à concorrência dos UGC e demais mídias.

Neste estudo, o contexto para a aplicação do UGC é o setor de aeroportos no Brasil. O setor de aeroportos é definido como serviços internacionais. O Brasil tem alguma tradição em obras de aeroportos, mas não na concessão de terminais. Após a compra de $70 \%$ da prestadora norte-americana de serviços aeroportuários TAS (Total Airport Services), pela CCR, por 21,7 milhões de dólares, em outubro de 2015 (CCR, 2015). Desde 2011, a CCR comprou fatias em administradoras de aeroportos no Equador, na Costa Rica e Curaçao. Para o setor de serviços a aquisição (operação greenfield) envolve menos risco. São prestados pela TAS, entre outros, serviços a passageiros, ou seja, check-in e emissão de bilhetes, serviços de partida, chegada (como direcionamento de passageiros em conexão) e processamento e manuseio de bagagem. Alguns estão entre os mais movimentados do mundo: Chicago O'Hare (ORD), Los Angeles (LAX), San Francisco (SFO), Atlanta Hartsfield (ATL) e Newark Liberty (EWR). Representa um momento único, para multinacionais brasileiras, atuarem em serviços globais como aeroportos.

METODOLOGIA

Analisam-se os comentários de usuários do GRU quanto ao conteúdo gerado pelo usuário (User-Generated Content - UGC), que apesar das críticas recebidas (equidade, qualidade, privacidade e propriedade intelectual), é a 
matéria prima para análise por meio de algoritmos de analítica de negócios e bases denominadas Big Data. Utiliza-se na pesquisa uma base de respondentes com 27 comentários (oito diferentes nacionalidades) com recorte temporal entre 07/11/2011 e 19/05/2014, cujos comentários constituem o corpus desta pesquisa (Krippendorff, 1989; Bauer \& Gaskell, 2002; Bardin, 2011).

Os comentários são extraídos de uma pesquisa de consulta pública disponível na base de dados da Skytrax ${ }^{1}$. A escolha do período para a pesquisa é anterior a Copa de 2014, por alguns principais motivos: A quantidade total de comentários acrescidos durante e após a Copa de 2014, até a data mais recente da pesquisa (05/02/2016) é de 13 comentários. Nove até 26/01/2015, com as três variáveis consideradas neste estudo.

A partir de 28/06/2015 ocorre uma mudança na metodologia da base de dados. São avaliadas seis novas variáveis, a saber:signs\&directions, seating, food\&beverages, shopping, wifi services e airport staff, que antes estavam incorporadas em facilities e queuing, apesar destas ainda permanecerem como variáveis.

Para considerar um estudo comparativo do contexto (GRU), antes e após a Copa do Mundo de 2014, há um número inferior de comentários, há a mudança de metodologia em 2015 que impossibilita a extração do que corresponderia às variáveis facilities e queuing (distribuídas nas seis novas variáveis), além de contemplar muitos dados faltantes, o que poderia ser prejudicial principalmente para a análise estatística. Por estes motivos opta-se pelo recorte temporal, que coincidentemente abrange o período da finalização da reforma do GRU para a Copa do Mundo 2014, e a transição do modelo de gestão público para privado, no setor aeroportuário do Brasil.

Justifica-se o tamanho da amostra 1) pelo recorte temporal; 2) pelo fato do questionário ser respondido de forma espontânea (ou orgânica na linguagem da mídia social), isto é, sem intervenção profissional (Shirky, 2008; Kaplan \& Haenlein, 2010) e sem nenhuma ação motivacional realizada pelo aeroporto, uma das características relevantes do UGC; 3) e pelo objetivo da pesquisa em analisar comentários de usuários (para avaliar serviços e a importância em usar a linguagem como método de análise), sendo o GRU um contexto (ambiente). 
Emprega-se a análise de conteúdo, a categorização (sim recomendam ou não recomendam o aeroporto), indicadores (frequência de palavras e significado no contexto) e variáveis independentes. Cada usuário respondeu a variáveis como: Dependentes: Ranking (escala de 1-10) e se recomenda o aeroporto (sim/não). Independentes: Tempo de espera na fila (escala de 1-5), limpeza (escala de 1-5), Instalações (escala de 1-5).

A análise de conteúdo (Rossi, Serralvo \& João, 2014) é uma metodologia aplicável em ciências sociais que possibilita o estudo de mensagens textuais. Por intermédio de processos técnicos, tais como: a análise do discurso; disposição e frequência de termos utilizados (palavras); e referências e construções linguísticas, o investigador busca compreender a mensagem transmitida. Integrando o objeto de estudo (comentários textuais) aos objetivos propostos neste artigo, referentes à opinião e à recomendação ou não do GRU por seus usuários, justifica-se a escolha da análise de conteúdo como principal metodologia, complementada por análises estatísticas.

Krippendorff (1989) aponta que a análise de conteúdo é uma técnica de pesquisa para fazer inferências replicáveis e válidas a partir de dados em um determinado contexto. Bardin (2011) e Richardson (1999) consideram que a análise de conteúdo é um conjunto de técnicas interpretativas de análise de comunicação sustentadas por processos técnicos de validação. Desta forma são identificados os principais recursos para a aplicação da metodologia, o que inclui a constituição da unidade de registro (ou análise) designada de corpus, definição das categorias e indicadores.

De acordo com Krippendorff (1990), Bauer e Gaskell (2002) e Bardin (2011), o corpus é o conjunto de documentos que são submetidos à análise, onde é estabelecida a unidade de registro e determinado o período de coleta de dados. Neste estudo, o conjunto dos comentários escritos pelos usuários que frequentam o GRU compõem o corpus para análise e cada comentário escrito representa a unidade de análise.

Após definir o corpus da análise, é necessário determinar as categorias. Para Bardin (2011) as categorias agrupam elementos que possuem características comuns sendo quatro os critérios de classificação sugeridos pela autora: semântico, sintético, léxico e/ou expressivo (tabela 1). 
Tabela 1 - Critérios de classificação para categorias

\begin{tabular}{|c|l|l|}
\hline CRITÉRIOS & \multicolumn{1}{|c|}{ DESCRIÇÃO } & \multicolumn{1}{|c|}{ UNIDADES } \\
\hline \hline SEMÂNTICO & $\begin{array}{l}\text { Pode ser identificado por meio da presença ou ausência e } \\
\text { frequência de elementos ou unidades de registro }\end{array}$ & $\begin{array}{l}\text { Temas, Palavras } \\
\text { ou outras } \\
\text { unidades }\end{array}$ \\
\hline SINTÁTICO & Serve para medir aintensidade da mensagem & $\begin{array}{l}\text { Verbos, Adjetivos } \\
\text { e Pronomes }\end{array}$ \\
\hline LÉXICO & $\begin{array}{l}\text { Informa o sentido e significado das palavras e aponta a } \\
\text { direção (favorável, neutra ou desfavorável) }\end{array}$ & Palavras \\
\hline EXPRESSIVO & Identifica as variações na escrita ou linguagem & $\begin{array}{l}\text { Linguagem e a } \\
\text { Escrita }\end{array}$ \\
\hline
\end{tabular}

Fonte: Adaptado de Bardin (2011)

Seguindo o critério léxico (tabela 1), o princípio de qualidade sugerido por Bardin (2011) e a realização de inferências replicáveis e válidas de Krippendorff (1989), são definidas duas categorias: Categoria Sim (recomenda o GRU) e a Categoria Não (não recomenda). As categorias estão diretamente relacionadas com a pontuação (ranking) auferida ao GRU (tabela 2).

Tabela 2 - Pontuação para recomendação ou não do aeroporto

\begin{tabular}{|c|l|c|c|}
\hline $\begin{array}{c}\text { QTD DE } \\
\text { COMENTÁRIOS }\end{array}$ & \multicolumn{1}{|c|}{ PAÍSES e ID CORRESPONDENTES } & $\begin{array}{c}\text { PONTUAÇÃo } \\
\text { RANKING }\end{array}$ & $\begin{array}{c}\text { RECOMENDA } \\
\text { Recommended }\end{array}$ \\
\hline \hline $\mathbf{2}$ & Reino Unido (1 e 11) & 7 & Yes \\
\hline $\mathbf{2}$ & Suíça (5) e Brasil (10) & 6 & Yes \\
\hline \hline $\mathbf{4}$ & Subtotal dos Comentários - Média da pontuação & $\mathbf{6 , 5}$ & YES \\
\hline \hline $\mathbf{2}$ & Brasil (8) e India (13) & 5 & No \\
\hline $\mathbf{2}$ & Reino Unido (9) e Brasil (14) & 4 & No \\
\hline $\mathbf{6}$ & $\begin{array}{l}\text { Reino Unido (2), Austrália (3), Canadá (18), Brasil (20 } \\
\text { e 25) e Estados Unidos (22) }\end{array}$ & 3 & No \\
\hline $\mathbf{9}$ & $\begin{array}{l}\text { Brasil (4, 7 e 21), Estados Unidos (6 e 15), Reino } \\
\text { Unido (12 e 23), Canada (19) e Suécia (26) }\end{array}$ & 2 & No \\
\hline $\mathbf{3}$ & Brasil (16 e 17) e Reino Unido (24) & 1 & No \\
\hline \hline $\mathbf{2 2}$ & Subtotal dos Comentários - Média da pontuação & $\mathbf{3}$ & NO \\
\hline $\mathbf{2 6}$ & Total & & \\
\hline
\end{tabular}

OBS: Os números entre parênteses representam o ID (código de identificação) do entrevistado.

Na tabela 2 nota-se que apenas quatro comentários, do total de 26, são de usuários que recomendam o GRU (categoria Sim), recebendo pontuação maior ou igual a seis, que representa a nota de corte para este critério. Uma pontuação igual ou inferior a cinco indica que o usuário está insatisfeito com os serviços oferecido no aeroporto e não o recomenda (Categoria Não). Considerando-se a nacionalidade dos entrevistados os brasileiros tendem a auferir pontuações negativas (iguais ou inferiores a três pontos - tabela 2). 
Na sequência são estabelecidos os indicadores (palavras), cujos critérios adotados são: 1) a frequência das palavras: repete-se seu uso por, no mínimo, três vezes (considerando-se o conjunto das 26 entrevistas), excluindo-se os pronomes, verbos e preposições e agrupando-se palavras singulares com seu plural correspondente; e 2) o significado das mesmas dentro do contexto em análise. Para tanto é utilizado o software de análise de texto TextStat, por ser de livre domínio, permitir facilidade de acesso e possibilidade de replicação dos resultados.

Os indicadores estão relacionados às categorias e às variáveis de análise da pesquisa: tempo de espera (queuing), instalações (facilities) e limpeza (cleanline). As variáveis referem-se às condições de uso do aeroporto (serviços estruturais oferecidos). A pontuação auferida a cada uma delas influencia o grau de recomendação ou não do aeroporto pelo usuário: Categoria Sim e Categoria Não.

Outra análise é utilizada em complementação aos procedimentos metodológicos: análise estatística. A análise estatística verifica a correlação entre as variáveis e o impacto em relação à recomendação, utilizando-se regressões para comparação de variáveis independentes (figura 1).

Figura 1 - Design da análise estatística

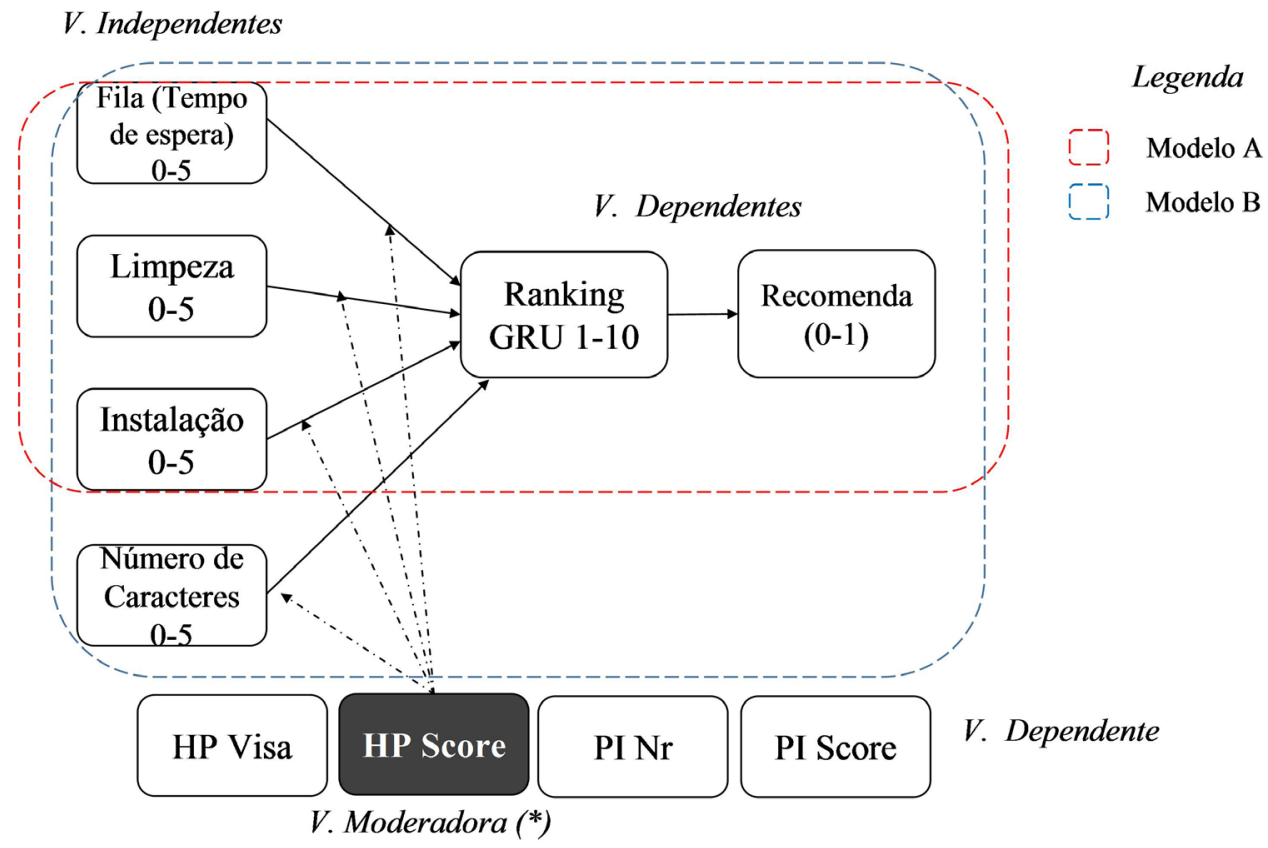

(*) Após correlação, variável moderadora reduzida à HP Score 
Para a correlação são consideradas quatro variáveis moderadoras (HP_Visa, HP_Score, PI_Nr e PI_Score) definidas por rankings de referência do setor: Henley \& Partners Visa Restrictions Index (HP) que analisa há dez anos o regulamento de vistos de todos os países e territórios; e a Passport Index (PI), que coleta, mostra e classifica passaportes do mundo todo. Estas organizações adotam dois indicadores: Visa que é o índice definido por um ranking global de países de acordo com a liberdade de viagens que os seus cidadãos possuem; e o Score que representa o número de países e territórios que podem ser inseridos sem visto por um cidadão do respectivo país. Realizados Correlação de Pearson verificou-se correlações entre 0,991 e 0,999. A seguir foram efetuados os diagnósticos de colinearidade e confirmado pela regressão (VIF < 10) adotouse unicamente o HP_Score tanto para o modelo A como o modelo B.

Duas rodadas foram realizadas na regressão, definindo-se assim os dois primeiros modelos para o design da pesquisa (figura 1). No Modelo A, analisamse as variáveis independentes: tempo de espera em fila (queuing), instalações (facilities) e limpeza (cleanline); e no Modelo B insere-se a variável independente número de caracteres.

\section{ANÁLISE DOS RESULTADOS}

Para os comentários positivos, significa que a situação do GRU não é a melhor, segundo os padrões internacionais, é aceitável, porém seguida de críticas. Para os comentários negativos, a não aprovação das condições do GRU (rejeição), por seus usuários. Os brasileiros da amostra são mais críticos que os estrangeiros. As variáveis de maior impacto são: tempo de espera nas filas e instalações. Limpeza é menos relevante.

No levantamento inicial definido para este estudo, utiliza-se o aplicativo TextStat a fim de quantificar algumas unidades pertinentes a mensagem escrita. Considerando-se o conjunto das 26 entrevistas coletadas, no período de dois anos e sete meses (início em 07/11/2011 e término em 18/05/2014), pode-se determinar que o universo de análise contenha: 17.894 caracteres impressos (incluindo a quantidade de espaços que corresponde a 3.161); 3.254 palavras 
distribuídas em 188 sentenças, sendo que o número médio de palavras por sentença é de 17,31. Estes dados são mais explorados na análise estatística.

O segundo recorte para a análise dos resultados segue o critério da categorização, separando-se os comentários em dois grandes blocos de mensagens: Categoria Sim com 529 palavras, distribuídas em quatro comentários favoráveis; e Categoria Não contendo 2725 palavras em um total de 22 entrevistados que são desfavoráveis à recomendação do aeroporto. A etapa seguinte é a definição dos indicadores (palavras) para extrair o sentido e o significado dos termos inseridos no contexto, de acordo com a forma de análise de conteúdo adotada neste estudo: léxica.

Por intermédio da análise da nuvem de palavras é possível notar a frequência e a utilização dos indicadores (palavras) pelo destaque visual (representado pelo tamanho da fonte) que os mesmos obtêm dentro da nuvem, tornando perceptível a sua imediata identificação.

Para a Categoria Sim são identificados os termos utilizados nos comentários favoráveis ao GRU, excluídas algumas palavras que eventualmente possam comprometer a análise dos resultados (por exemplo: Airport, International), agrupados os sinônimos e a forma singular e plural das palavras, resultando na nuvem de palavras Categoria Sim (figura 2).

Figura 2 - Nuvem de Palavras Categoria Sim (comentários favoráveis)

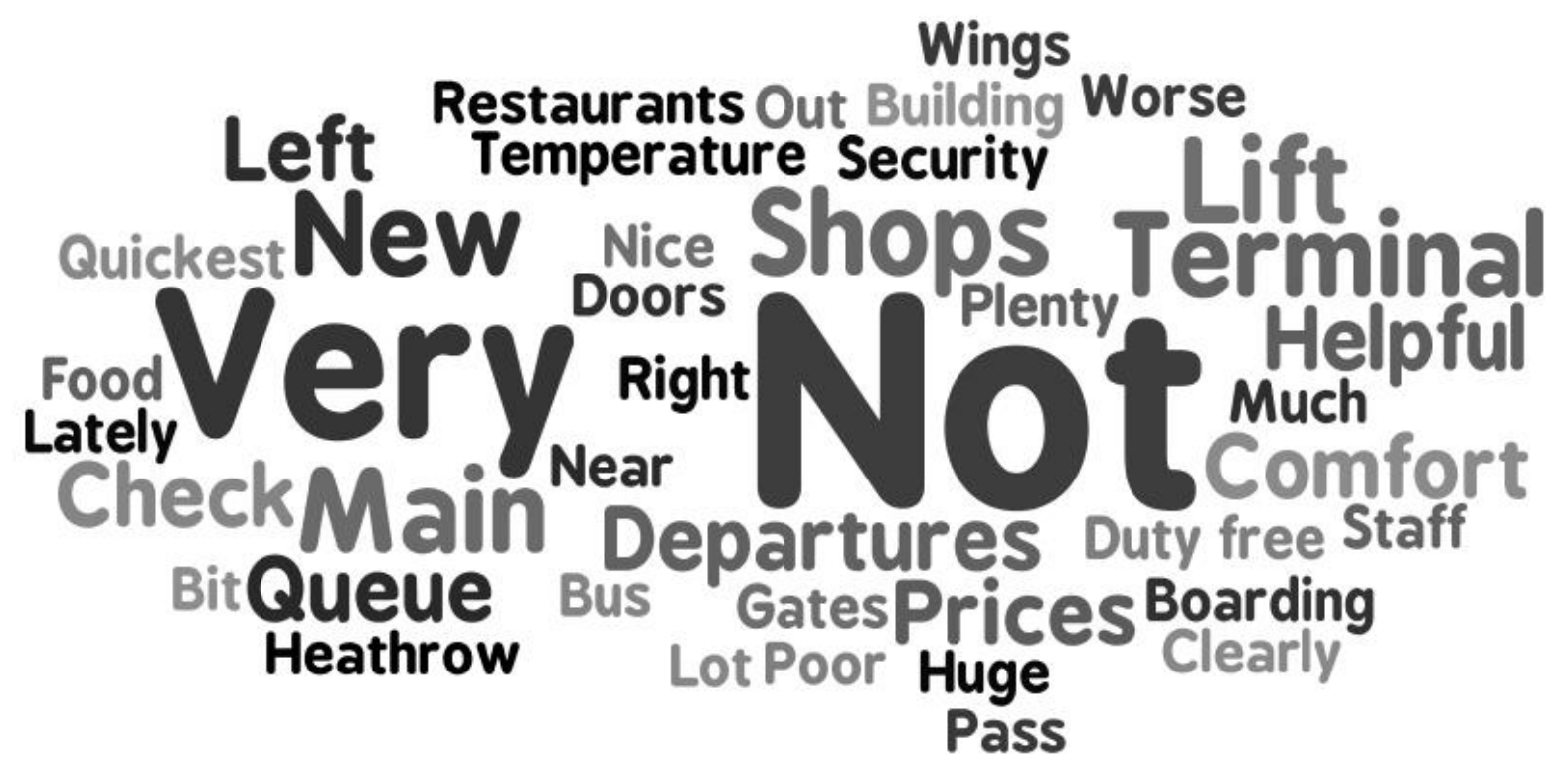

As palavras analisadas (tabela 3) são as que obtiveram maior destaque na nuvem (figura 2): Not, Very, Lift, Terminal, New, Shops, Main, Prices e Comfort. 
Apesar de serem comentários positivos, há uma forte presença da palavra Not. Dentro do contexto dos comentários, o Not significa que a situação do GRU não é a melhor para os padrões internacionais, mas é aceitável, conforme ilustrado nos trechos extraídos: "...Its not the quickest immigration queue I've been in, but by no means the worst... (ID 1- Reino Unido)" e "...staff are not very efficient but very nice... (ID 5 - Suíça)".

Tabela 3 - Análise dos indicadores da Categoria Sim

\begin{tabular}{|c|c|c|c|}
\hline $\begin{array}{c}\text { INDICADORES } \\
\text { (palavras ou } \\
\text { termos) }\end{array}$ & $\begin{array}{c}\text { FREQUÊNCIA } \\
\text { da PALAVRA } \\
\text { (nos } \\
\text { comentários) }\end{array}$ & SENTIDO e SIGNIFICADO DENTRO DO CONTEXTO & VARIÁVEIS \\
\hline NOT & 8 & $\begin{array}{l}\text { Na maioria das vezes o Not refere-se ao tempo de espera nos } \\
\text { processos administrativos (queuing), tais como: imigração, check } \\
\text { in e preparo do staff; ou relativos às instalações (facilities): } \\
\text { restaurantes e lojas, e temperatura ambiente. "There's not a huge } \\
\text { range of shops before departures, but we had a nice lunch at the } \\
\text { buffet restaurant... (ID 1- Reino Unido)"; “...Staff are not very } \\
\text { efficient but very nice... (ID } 5 \text { - Suiça) ". O termo também enfatiza } \\
\text { que apesar de não ser a situação ideal, não é a pior, isto é, } \\
\text { existem aeroportos em piores condições que o GRU na opinião } \\
\text { de alguns entrevistados. }\end{array}$ & $\begin{array}{l}\text { Queuing, } \\
\text { Facilities }\end{array}$ \\
\hline VERY & 7 & $\begin{array}{l}\text { Está associado à prestação de serviços de taxi e staff (queuing) e } \\
\text { instalações ( facilities ). O destaque fica para o comentário que } \\
\text { retratou uma experiência positiva sobre tempo de espera "... I had } \\
6 \text { hours wait and it passed very comfortably ... (ID } 11 \text { - Reino } \\
\text { Unido) " }\end{array}$ & $\begin{array}{l}\text { Queuing, } \\
\text { Facilities }\end{array}$ \\
\hline LIFT & 4 & $\begin{array}{l}\text { Esta palavra é utilizada apenas em um comentário e serve como } \\
\text { ponto de referência ".... There is an information desk near the lift - } \\
\text { staff very helpful and speak English ... (ID } 11 \text { Reino Unido) }\end{array}$ & $N / A$ \\
\hline TERMINAL & 4 & $\begin{array}{l}\text { Esta palavra é utilizada apenas em um comentário e está } \\
\text { associada à facilities, considerada como melhoria nas } \\
\text { instalações do terminal } 4 \text {, inaugurado em fevereiro de } 2012 . " \text { ".... } \\
\text { Many quick improvements have been done lately, like new } \\
\text { restrooms, new restaurants and a real upgrade in terminal signs } \\
\ldots \text {.. (ID } 10 \text { Brasil)" }\end{array}$ & Facilities \\
\hline NEW & 4 & $\begin{array}{l}\text { A palavra new aparece em apenas um comentário e é designada } \\
\text { para novo terminal e novas lojas, ou seja, melhoria nas } \\
\text { instalações. } \\
\text { ".... Many quick improvements have been done lately, like new } \\
\text { restrooms, new restaurants and a real upgrade in terminal signs } \\
\ldots \text { (ID } 10 \text { Brasil)" }\end{array}$ & Facilities \\
\hline SHOP(S) & 4 & $\begin{array}{l}\text { A palavra está relacionada a quantidade de lojas, portanto à } \\
\text { facilities. Somente um comentário (ID } 11 \text { - Reino Unido) afirma ser } \\
\text { a quantidade suficiente "... Plenty of seating (although I agree the } \\
\text { seats could be more comfortable) and sufficient food outlets and } \\
\text { duty free shops... (ID } 11 \text { - Reino Unido) " }\end{array}$ & Facilities \\
\hline MAIN & 4 & $\begin{array}{l}\text { Esta palavra está associada à facilities, porém na percepção dos } \\
\text { usuários não representa um comentário positivo e sim um alerta } \\
\text { para estabelecer melhorias nas instalações do aeroporto ".... } \\
\text { Guarulhos Airport main building still looks a bit dated overall and it } \\
\text { was really crowded. ... (ID } 10 \text { Brasil)" e "... My main issue here is } \\
\text { the poor signage... (ID } 11 \text { - Reino Unido)" }\end{array}$ & Facilities \\
\hline $\operatorname{PRICE}(S)$ & 4 & $\begin{array}{l}\text { Price refere-se aos preços altos praticados nas lojas e } \\
\text { restaurantes locais, porém estão de acordo com o padrão de } \\
\text { preços de aeroportos internacionais "...The food is overpriced, but } \\
\text { no different from other airports I have visited.... (ID } 11 \text { - Reino } \\
\text { Unido)" e ".... The prices might be a bit high by Brazilian } \\
\text { standards..." (ID 1 - Reino Unido)" }\end{array}$ & Facilities \\
\hline COMFORT & 3 & $\begin{array}{l}\text { Nos dois comentários onde a palavra é citada há referência sobre } \\
\text { melhorias neste quesito "... Hope to see improvements, especially } \\
\text { in comfort ... (ID } 10 \text { - Brasil)" e " ... although I agree the seats could } \\
\text { be more comfortable ... (ID } 11 \text { - Reino Unido)". Apenas um usuário } \\
\text { sente-se confortável com as instalações do aeroporto: "... I had } 6 \\
\text { hours wait and it passed very comfortably... (ID } 11 \text { - Reino Unido) " }\end{array}$ & Facilities \\
\hline
\end{tabular}


Após extrair os termos da nuvem de palavras (figura 2) e preparar a tabela 3, identifica-se que a palavra Lift é utilizada para designar um ponto de referência dentro do aeroporto, e neste contexto não representa um indicador para análise de conteúdo, portanto deve ser desconsiderado da análise.

Por meio dos comentários selecionados na tabela 3 pode-se notar que a grande maioria contém observações referentes às instalações (facilities). Apesar de representar a Categoria Sim, a maioria dos comentários não apresenta um elogio ao GRU, mas uma aceitação das condições e, às vezes, uma crítica "... Far away from downtown ... It isn't clean at all, ..., there's very few shops ..." (ID 5 - Suíça). Esta prerrogativa corrobora com a grade de notas auferidas pelos usuários. Numa escala de zero a dez, as maiores notas recebidas pelo GRU estão muito próximas do limite (nota de corte), correspondendo às pontuações seis e sete.

Na figura 3 são apresentadas as palavras que compõem a Categoria Não. Estas são extraídas dos comentários não-favoráveis à indicação do GRU, passam por um processo de análise e síntese, onde são excluídos os termos que podem distorcer a análise, tais como: Airport, International, entre outros, agrupando-se os sinônimos e as formas singular e plural das palavras.

Figura 3 - Nuvem de Palavras Categoria Não (comentários desfavoráveis)

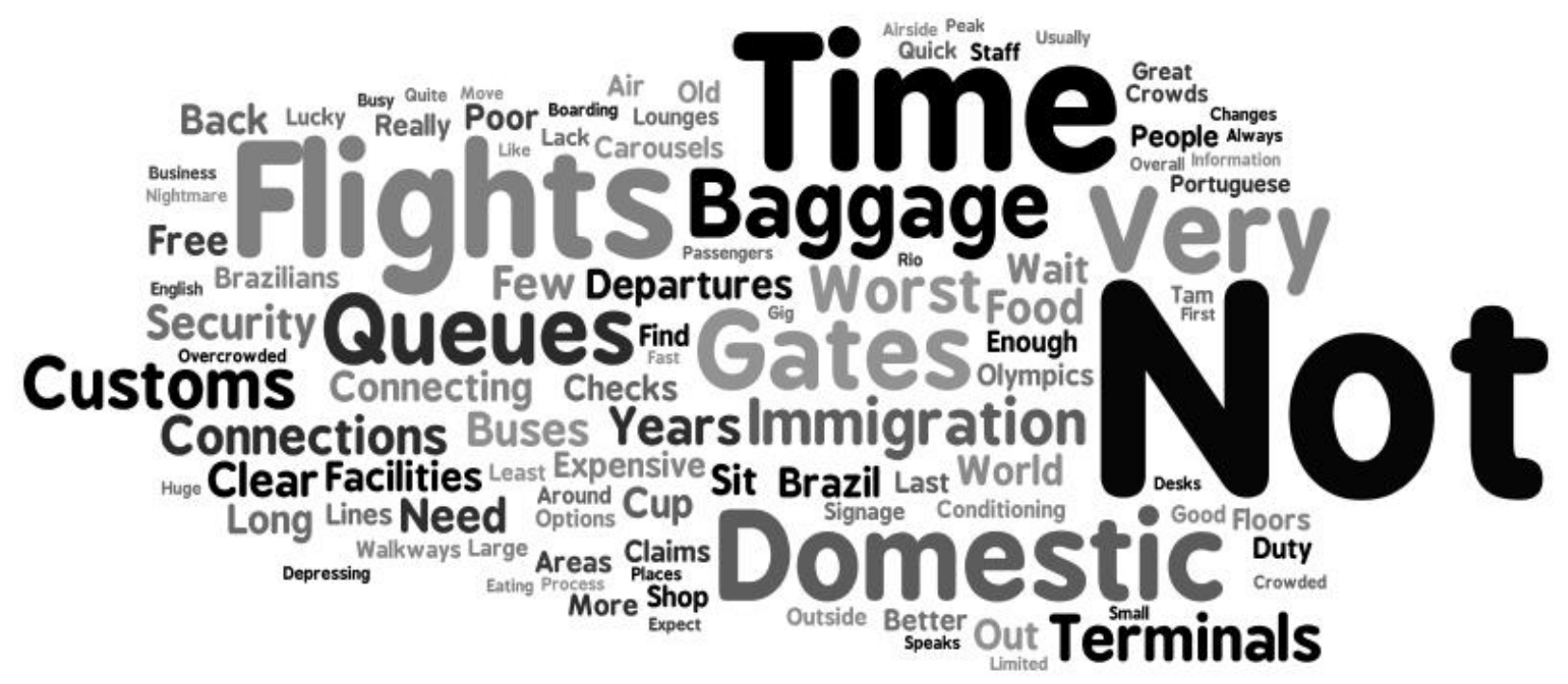

As palavras, ou grupo de palavras analisadas (tabela 4) são as de uso mais frequente nos comentários, e por isso obtêm destaque na nuvem de palavras (figura 3): Not, Time/Hour(s)/Minutes, Fligt(s), Domestic, Very, Gate(s), Queue(s), Baggage/Luggage e Worse/Worst. Através da visualização das palavras na nuvem (figura 3) nota-se que o termo Not apresenta uma correlação à categoria 
que representa: Categoria Não, ou seja, a forte presença da palavra Not, neste contexto, significa a não aprovação das condições do GRU por seus usuários.

\section{Tabela 4 - Análise dos indicadores da Categoria Não}

\begin{tabular}{|c|c|c|c|}
\hline $\begin{array}{l}\text { INDICADORES } \\
\text { (palavras ou } \\
\text { termos) }\end{array}$ & $\begin{array}{l}\text { FREQUÊNCIA } \\
\text { da PALAVRA } \\
\text { (nos } \\
\text { comentários) }\end{array}$ & SENTIDO e SIGNIFICADO DENTRO DO CONTEXTO & VARIÁVEIS \\
\hline NOT & 35 & $\begin{array}{l}\text { O Not, presente em } 11 \text { dos } 26 \text { comentários, representa a insatisfação } \\
\text { dos usuários em relação às condições gerais do GRU "... They simply } \\
\text { do not have the facilities. (ID } 19 \text { Canada) ..." "...They are not ready for } \\
\text { World Cup or the Olympics. ... (ID } 6 \text { EUA) ..." além de apontar a } \\
\text { insulficiência de alguns itens, tais como: cadeiras, mais opções de } \\
\text { comida, falta de sinalização ou dificudade em compreende-la etc. "... Not } \\
\text { enough seats. ... (ID } 19 \text { Canada)". “..., there are very few places to eat, } \\
\text { expensive food and are not good but very busy. ... (ID } 18 \text { Canada)". "..., I } \\
\text { am not sure if they announced it or not, even if they had, I would not have } \\
\text { understood. ... (ID } 13 \text { İndia)". }\end{array}$ & Facilities \\
\hline $\begin{array}{l}\text { TIME, HOUR(S), } \\
\text { MINUTES }\end{array}$ & 30 & $\begin{array}{l}\text { Referem-se ao tempo de espera (filas) "If you're arriving at peak time, be } \\
\text { prepared to wait at least } 1 \text { and a half hours to clear immigration and get } \\
\text { your luggage and clear customs. (ID } 8 \text { Brasil)". }\end{array}$ & Queuing \\
\hline FLIGT(S) & (i) & $\begin{array}{l}\text { Palavra presente em } 15 \text { comentários e refere-se às conexões de voos: } \\
\text { falta de sinalização e informação, falta de espaço fisico para espera nas } \\
\text { filas e nas esteiras (desorganização na distribuição das bagagens). } \\
\text { "Not enough gates so we have to take a bus to get to a connecting flight... } \\
\text { (ID } 6 \text { EUA) ", "... usually they change the gate for your flight randomly } \\
\text { every } 30 \text { minutes of so... (ID } 22 \text { EUA)", "Facilities still very limited - had } \\
\text { multiple flights luggage on } 1 \text { carousel ... (ID } 3 \text { Austrália)", "They } \\
\text { crammed } 3 \text { large flights into a small holding area ... (ID 19 Canada)" }\end{array}$ & $\begin{array}{l}\text { Queuing, } \\
\text { Facilities }\end{array}$ \\
\hline DOMESTIC & 21 & $\begin{array}{l}\text { São as conexões e voos domésticos. "... Coming back for domestic } \\
\text { transfer, quite boring transit but did not need to re-enter security. ... (ID } 23 \\
\text { Reino Unido)" O ID } 9 \text { (Reino Unido) explica o processo longo e } \\
\text { complicado das conexões. }\end{array}$ & Facilities \\
\hline VERY & i & $\begin{array}{l}\text { Apesar da palavra very, em tradução livre, ser muito, no contexto ela } \\
\text { associa-se ao termo pouco, evidenciando a insuficiência de recursos } \\
\text { (lojas, restaurantes) e condições precárias do GRU: "Very few places to } \\
\text { buy food and what food that is on sale is very expensive even by } \\
\text { international airport standards ... (ID } 12 \text { Reino Unido)", "... It is very old, } \\
\text { outdated, and in need of desperate upgrading ... (ID } 15 \text { EUA)". } \\
\text { No entanto há comentários positivos referente às filas "...I found the non- } \\
\text { Brazilian queue very quick .... (ID } 9 \text { Reino Unido)" e "... Customs went } \\
\text { very fast, but I heard it can take longer in peak season... (ID } 18 \text { Canada)". }\end{array}$ & $\begin{array}{l}\text { Queuing, } \\
\text { Facilities }\end{array}$ \\
\hline GATE(S) & ind & $\begin{array}{l}\text { As reclamações referentes aos gates são: pouca quantidade, mudança } \\
\text { constante, aglomeração de pessoas e a convergência de vários voos } \\
\text { por um mesmo portão. "..., and downstairs to a crowded departure gate } \\
\text { serving two flights. ... (ID } 4 \text { Brasil)", "... where flights of 200-350 people } \\
\text { leave at a single gate, there may only be } 40-50 \text { seats ... (ID } 15 \text { EUA)", } \\
\text { "There are no gates: you have to take buses to the aircraft's ... (ID } 16 \\
\text { Brasil)", "Queues at bus gates all mixed up, very easy to get on the wrong } \\
\text { flight ... (ID } 26 \text { Suécia)" }\end{array}$ & $\begin{array}{l}\text { Queuing, } \\
\text { Facilities }\end{array}$ \\
\hline QUEUE(S) & 16 & $\begin{array}{l}\text { As filas geralmente são longas, desorganizadas e com longo tempo de } \\
\text { espera, . "... check in usually a mess, immigration and customs queues } \\
\text { ok if you are lucky ....(ID } 8 \text { Brasil)" e "... At the airport there are always } \\
\text { huge queues for everything ... (ID } 16 \text { Brasill)'. }\end{array}$ & $\begin{array}{l}\text { Queuing, } \\
\text { Facilities }\end{array}$ \\
\hline $\begin{array}{l}\text { BAGGAGE e } \\
\text { LUGGAGE }\end{array}$ & 12 & $\begin{array}{l}\text { As palavras baggage e luggage referem-se às malas, porém no } \\
\text { contexto representam a desorganização, tempo de espera e poucas } \\
\text { esteiras disponiveis para retirada. O comentário de um brasileiro } \\
\text { resume a opinião dos demais usuários: "... then baggage claim and } \\
\text { customs are your surprise nightmare... (ID } 25 \text { Brasil)". }\end{array}$ & $\begin{array}{l}\text { Queuing, } \\
\text { Facilities }\end{array}$ \\
\hline $\begin{array}{c}\text { WORSE e } \\
\text { WORST }\end{array}$ & 11 & $\begin{array}{l}\text { Nos sete comentários em que as palavras aparecem, apenas um } \\
\text { afirma que o GRU não é o pior aeroporto que o usuário frequenta, mas } \\
\text { não significa que seja recomendável. "... Not the worst airport I've been } \\
\text { to, but I can't even conceive it being ready for the crowds expected to } \\
\text { descent for the World Cup ... (ID } 20 \text { Brasil)" }\end{array}$ & Facilities \\
\hline
\end{tabular}

Por intermédio da análise da tabela 4 nota-se que a opinião da maioria dos entrevistados (22 indivíduos) é desfavorável ao GRU, portanto insatisfeitos com as condições gerais apresentadas no aeroporto, sentimento este resumido por um dos comentários extraídos: "There's really nothing good to be said about the place - sorry. (ID 7 Brasil)". 
Outro aspecto importante é que os brasileiros tendem a ser mais críticos em suas avaliações ao aeroporto, do que os estrangeiros, fato este que pode ser observado por alguns trechos extraídos de seus comentários: "It's old, bad food, small, overcrowded and when you arrive for customs you have the real idea of the nightmare you are entering. No queue and no staff to organize the mess. If you can avoid it, do. (ID 21 Brasil)" e "I have the displeasure to make stopovers in São Paulo every year, and it seems to get worse every time. (ID 16 Brasil)".

Pelo baixo índice de recomendação de usuários ao GRU (apenas quatro comentários são favoráveis), a forte presença da palavra Not nas duas categorias em análise, pode-se concluir que o aeroporto não agrada seus usuários e também que é necessário investir em melhorias principalmente nas suas instalações para tornar o GRU um lugar mais agradável.

De acordo com Lovelock e Wirtz (2010) a qualidade do serviço prestado ao usuário do GRU enquadra-se no Ciclo da Mediocridade, comum em organizações burocráticas, em mercados monopolizados e/ou ainda muito regulamentados. No caso específico do GRU, a maioria dos usuários manifesta sua insatisfação à qualidade do serviço prestado, através de constantes e repetidas reclamações, porém tendem a não ser cooperativos em soluções ou sugestões para que o aeroporto melhore a qualidade de seus serviços. Este aspecto pode ser uma das justificativas para a quantidade de questionários coletados em dois anos e meio, antes da Copa e da nova gestão e pela ênfase às críticas (divulgação e compartilhamento das más experiências).

Tanto nos comentários favoráveis, como nos desfavoráveis, as variáveis mais impactantes, isto é, de maior peso na opinião dos usuários, são referentes às instalações (facilities) e tempo de espera (queuing), sendo que a avaliação para o item limpeza (cleanline) é bem menos relevante e aparece somente em quatro comentários, sendo que dois deles consideram o GRU um lugar limpo: "Only positive aspect is that Guarulhos airport is pretty clean and security has improved. (ID 17 Brasil)" e "The airport is very clean, which is good as you'll probably end up sitting on the floor due to the overcrowding and lack of facilities. (ID 22 Estados Unidos)"; e os outros dois afirmam ser o GRU um lugar sujo: "It isn't clean at all, ... (ID 5 Suíça)", e "Toilets not overly clean. ID 3 Austrália)".

A palavra Not fortemente presente nas duas categorias analisadas (Categoria Sim e Categoria Não) confirma e é coerente ao alto grau de rejeição dos usuários 
ao transitarem pelo aeroporto e, em sendo o GRU uma importante porta de entrada para o país, pode impactar negativamente a imagem do Brasil ou a escolha do país como um destino de turismo e lazer: I enjoy visiting Brazil, but when I think of what I have to endure to get there, I prefer to go elsewhere. (ID 6 Estados Unidos)".

Em complementação à análise de conteúdo, emprega-se a análise estatística que apresenta uma forte correlação (de Pearson) entre as variáveis moderadoras (Sig = 0,01) a saber: HP_Visa e HP_Score $(-0,999) ; P I \_N r$ e PI_Score $(-0,992)$; o que possibilitou a redução das quatro variáveis a uma de maior valor correlacional: HP_Score $(-0,999)$ confirmada por diagnóstico de colinearidade.

Definida a variável moderadora, roda-se a regressão para os dois modelos ( $A$ e B) e constata-se que a variável Número de Caracteres não altera os resultados $(B=0,000)$, sendo assim desprezada (Figura 4). Além disso, no Modelo $B, a$ significância (ANOVA) foi de 0,07 e no Modelo A foi de 0,00 (99\%).

Com teste de normalidade ANOVA com Sig $=0,000$ e $R^{2}=0,524$ a regressão aponta que a variável de maior valor é a instalações (facilities) $=+1,052$, seguida do tempo de espera em fila (queuing) $=+0,371$, e limpeza (cleanline) $=+0,181$ (figura 4).

Figura 4 - Design da pesquisa (Modelos A e B) com resultados estatísticos e Pesquisa Futura (Modelo C)

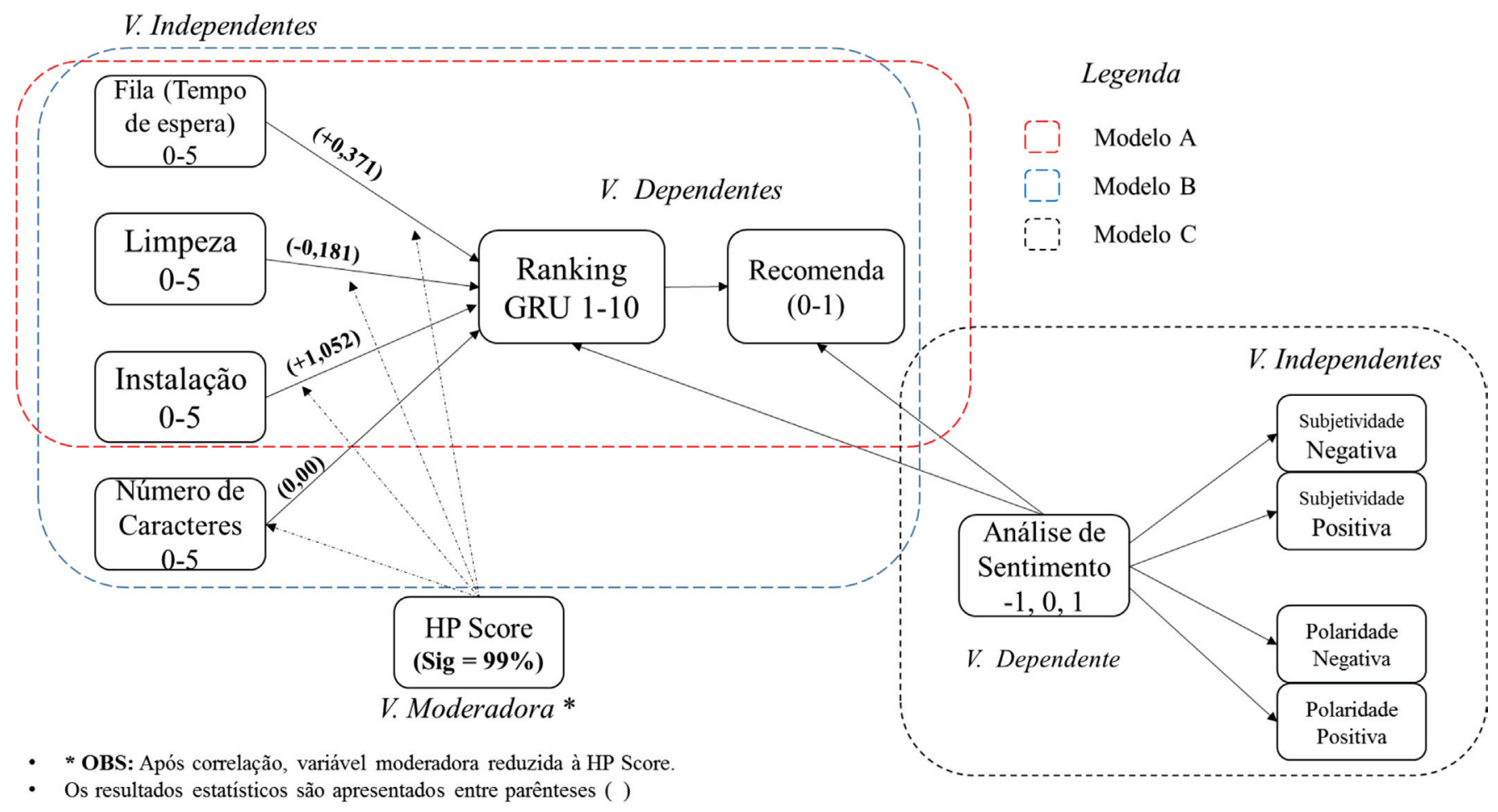


Tendo como base a aplicação dos Modelos A e B (Figura 4), verifica-se que os resultados estatísticos corroboram com os obtidos na análise de conteúdo, onde a variável de maior impacto nos comentários, tanto positivos, como negativos, é a instalações (facilities). Nota-se que a variável número de caractere $(0,000)$ não impacta os resultados, sendo assim desconsiderada da análise. O Modelo C é uma proposta de trabalho futuro e será visto nas considerações finais desta pesquisa.

\section{CONSIDERAÇÕES FINAIS}

Neste estudo reforça-se a importância de se utilizar metodologia de análise de conteúdo como ferramenta estratégica para a identificação de falhas em serviços assim como o aprimoramento do mesmo. Comentários gerados pelo usuário, ou seja, perspectiva dos usuários (service encounter) não é um tema novo em marketing de serviços. O que torna novo é a dinâmica criada pela utilização de ferramentas de tecnologia da informação e a geração de conteúdo que pode ser utilizado para a co-criação ou para a melhoria de qualidade ou redução de falhas em serviços. Tomando como exemplo um espaço público, que representa o aeroporto de Guarulhos, este estudo pode ser aplicado a diversos outros prestadores de serviços, ou avaliar o desempenho de um produto, sempre sob a ótica do consumidor.

Um dos pontos relevantes analisados na variável instalações (facilities) refere-se a falta de sinalização e informação, na sua maioria relacionadas às falhas decorrentes de respostas dos funcionários a problemas no sistema de prestação de serviço (Bitner, Booms \& Tetreault, 1990). Neste contexto, podem ser verificadas nos trechos extraídos da Categoria Não, nos comentários sobre Fight(s) e Gate(s) evidenciando o problema da influência da linguagem quando o pessoal de serviços e o cliente não falam o mesmo idioma (Holmqvist \& Grönroos, 2012) e o desafio para os gestores de recrutar profissionais de serviços com competência linguística e/ou multilíngues (Harzing \& Pudelko, 2013; Piekkari, Welch, Welch, Peltonen \& Vesa, 2013).

Ainda em questão às críticas dos usuários, destaca-se o Ciclo de Mediocridade de Lovelock e Wirtz (2010), no qual a maioria dos usuários manifesta sua 
insatisfação à qualidade do serviço prestado, porém não são cooperativos em soluções ou sugestões para que o aeroporto melhore a qualidade de seus serviços. Ou seja, a qualidade da prestação de serviços é uma via de mão dupla, construída em sintonia cliente e organização.

Estudos futuros podem contemplar um recorte temporal pós-reforma, que ampliou o aeroporto com a construção de mais um terminal. Recomenda-se verificar se a reforma e a mudança de modelo de gestão influenciam (ou não) a opinião dos usuários. Deve-se considerar as novas variáveis incorporadas na metodologia da base de dados (signs\&directions, seating, food\&beverages, shopping, wifi services e airport staff) e buscar novas bases ou aeroportos para comparação. Além disso, sugere-se incluir variáveis geradas a partir do texto do usuário como as encontradas em análise de sentimentos (sentiment analysis). A base de dados é equivalente a esta onde a partir do texto são geradas novas variáveis independentes, ou seja, análise de sentimento (positiva, neutra, negativa), subjetividade (positiva, negativa) e polaridade (positiva, negativa) conforme apresentada na figura 4 contra as variáveis dependentes lançadas pelo próprio consumidor (rankink e recomendação). Outras recomendações incluem mineração de dados, algoritmos como o Map Reduce, técnicas de identificação de padrão (fraudes em comentários, por exemplo) e outras técnicas de análise de Big Data além naturalmente do uso da linguagem em pesquisa que pode incluir análise de discurso, muito utilizada pelas ciências sociais e linguística. Dessa forma temse uma ferramenta de uso gerencial e estratégico, para o acompanhamento de serviços e, de certa forma, para a detecção de crises de imagem e de reputação.

\section{REFERÊNCIAS}

Bardin, L. (2011). Análise de conteúdo. São Paulo: Edições 70.

Bauer, M. W. \& Gaskell, (2002). G. Pesquisa qualitativa com texto, imagem e som: um manual prático. Petrópolis: Vozes.

Berry, L. L. (1980). Services Marketing Is Different. Business, 30(3), 24-29.

Bitner, M.J. (1990). Evaluating service encounters the effects of physical surroundings and employee responses. Journal of Marketing, 54(2), 69-82. 
Bitner, M.J., Booms, B.H. \& Tetreault, M.S. (1990). The Service Encounter: Diagnosing Favorable and Unfavorable Incidents. Journal of Marketing. 54(January 1990), 71-84.

Blackshaw, P. \& Nazzaro, M. (2006). Consumer-generated media (CGM) 101: Wordof-mouth in the age of the web-fortified consumer. New York: Nielsen BuzzMetrics. Disponível em < http://www.nielsen-online.com/downloads/us/ buzz/nbzm_wp_CGM101.pdf >. Acesso em 10 out. 2016.

CCR. (2015). CCR S.A. announces signature of agreement for the acquisition of Total Airport Services - TAS. Disponível em http://ri.ccr.com.br/grupoccr/web/mobile/conteudo_mobile. asp?tipo $=47361 \&$ id $=217710 \&$ idioma $=1 \&$ conta $=44$. Acesso em 5 out. 2015

Chase, R. B. \& Stewart, D.M. (1994). Make Your Service Full-Safe. Sloan Management Review. Spring 1994: 35-44.

Content Marketing Institute. (2016). 2015 Benchmarks, Budgets, and Trends - North America Disponível em http://contentmarketinginstitute.com/wp-content/uploads/2014/10/2015_ B2B_Research.pdf

Dennhardt, S. (2014). User-Generated Content and its Impact on Branding: How Users and Communities Create and Manage Brands in Social Media. ...Springer Gabler.

Fitzsimmons, J. A. \& Fitzsimmons, M. J. (2014) Administração de Serviços - Operações, Estratégia e Tecnologia da informação. Porto Alegre: McGraw-Hill Education e Bookman.

Grant, R. M. (2010). Contemporary Strategy Analysis. 7th ed. West Sussex. John Wiley $\&$ Sons.

Grönroos, C. (1990). Relationship approach to the marketing function in service contexts: the marketing and organizational behavior interface. Journal of Business Research, 20(1), 3-12.

Grönroos, C. (2008). Service logic revisited: who creates value? And who co-creates? European Business Review, 20(4), 298-314.

Grönroos, C. \& Voima, P. (2013). Critical service logic: making sense of value creation and co-creation. Journal of the Academy of Marketing Science, 41(2), 133-150.

Harzing, A. W. \& Pudelko, M. (2013). Language competencies, policies and practices in multinational corporations: a comprehensive review and comparison of Anglophone, Asian, Continental European and Nordic MNCs. Journal of World Business, 48(1), p. 87-97.

Hax, A.C. (2010). The Delta Model: Reinventing Your Business Strategy. New York: Springer.

Heidenreich, S., Wittkowski, K, Handrich, M. \& Falk, T. (2014). The dark side of customer cocreation: exploring the consequences of failed co-created services. Journal of the Academy of Marketing Science. 43(3), 279-296. 
Holmqvist, J. (2011). Consumer language preferences in service encounters: a cross-cultural perspective. Managing Service Quality, 21(2), 178-191.

Holmqvist, J. \& Grönroos, (2012). C. How does language matter for services? Challenges and propositions for service research. Journal of Service Research, 15, 430-442.

Holmqvist, J. \& Van Vaerenbergh, Y. (2013). Perceived importance of native language use: high-and low-involvement service encounters. Service Industries Journal, 33(15/16), 1659-71.

Kaplan, A. M.\& I Haenlein, M. (2010). Users of the world, unite! The challenges and opportunities of Social Media, Business Horizons, 53, 59-68.

Krippendorff, K. (1989). Content analysis. ScholaryCommons, Annenberg School for Communication, University of Pennsylvania, p. 402-408.

Krippendorff, K. (1990). Metodologia de analisis de contenido: teoria y práctica. Barcelona: Paidós.

Lovelock, C. \& Wirtz, J. (2010). Services Marketing: people, technology, strategy. New Jersey: Prentice Hall.

Luna, D. \& Peracchio, L. A. (2001). Moderators of language effects in advertising to bilinguals: a psycholinguistic approach. Journal of Consumer Research, 28(2), 284-95.

Payne, A.F., Storbacka, K \& Frow, P. (2008). Managing the co-creation of value. J. of the Acad. Mark. Sci. 36:83-96.

Piekkari, R.; Welch, D. E.; Welch, L. S.; Peltonen, J. P. \& Vesa, T. (2013). Translation behavior: An exploratory study within a service multinational. International Business Review, 22(5), 771-783.

Porter, M.E. (1980). Competitive Strategy: Techniques for Analyzing Industries and Competitors. New York: Free Press.

Porter, M.E. (1981). The contribution of industrial organization to strategic management, Academy of Management Review. 6(4), 609-20

Porter, M.E. (1985). Competitive Advantage: Creating and Sustaining Superior Performance New York: Free Press.

Porter, M.E. (1996). What is strategy?. Harvard Business Review, 96(6), 61-78

Puntoni, S.; De Langhe, B. \& Van Osselaer, S. M. J. (2009). Bilingualism and the emotional intensity of advertising language. Journal of Consumer Research, 35(6), 1012-25. 
Richardson, R. J., (1999). Pesquisa social: métodos e técnicas, São Paulo: Atlas.

Rossi, G.B., Serralvo, F.A. \& João, B.N. (2014). Análise de Conteúdo. Revista Brasileira de Marketing. 13(4), 39-48.

Salciuviene, L.; Ghauri, N.P.; Streder, R.S. \& De Mattos, C. (2010). Do brand names in a foreign language lead to different brand perceptions? Journal of Marketing Management, 26(1112), 1037-1056.

Shirky, C. (2008). Here comes everybody: the power of organizing without organizations. London: Penguin Books Ltd. Disponível em: < https://uniteyouthdublin.files.wordpress. com/2015/01/here_comes_everybody_power_of_organizing_without_organizations.pdf >. Acesso em 10 out. 2016.

Shostack, G. L. (1984). Designing services that deliver. Harvard Business Review, Jan-Feb, 1984, 133-139.

Sidershmukh, D.; Singh, J. \& Sabol, B. (2002). Consumer trust, value, and loyalty in relational exchanges. Journal of Marketing, 66, 15-37.

Smith, A.N., Fischer, E. \& Yongjian, C. (2012). How Does Brand-related User-generated Content Differ across YouTube, Facebook, and Twitter?. Journal of Interactive Marketing. 26(2), 102-113.

Spielmann, N. \& Delvert, M. (2014). Adapted or standardized copy: is non-cultural English the answer? Journal of Business Research, 67(4), 434-440.

Stewart, D. \& Chase, R. (1999). The impact of human error on delivering service quality. Production and Operations Management, 8(3), 240-263.

Surprenant, C.F. \& Solomon, M.R. (1987). Predictability and personalization in the service encounter. Journal of Marketing, 51(2), 73-80.

Treacy, M. \& Wiersema, F. (1995). The Discipline of Market Leaders - Choose Your Customers, Narrow Your Focus, Dominate Your Market. Reading. MA: Addison-Wesley.

Van Vaerenbergh, Y. \& Holmqvist, J. (2013). Speak my language if you want my money! Service language's influence on consumer tipping behavior. European Journal of Marketing, 47(8), 1276-1292. 
Van Vaerenbergh, Y. \& Holmqvist, J. (2014). Examining the relationship between language divergence and word-of-mouth intentions. Journal of Business Research, forthcoming, 67(8), 1601-1608.

Vargo, S. L. \& Lusch, R. F. (2004). Evolving to a new dominant logic for marketing. Journal of Marketing, 68, 1-17.

Xiang Z. \& Gretzel U. (2010) Role of social media in online travel information search. Tourism Management, 31, 179-188.

\section{Contribuição dos autores na construção do artigo} Guido: Revisão teórica, análise de conteúdo e conclusões. João: Revisão teórica, análise estatística e conclusões. 